\title{
9. MIDDLE EOCENE TO MIOCENE PLANKTONIC FORAMINIFERS FROM DEEP SEA DRILLING PROJECT SITES 608 AND 610, NORTHEASTERN ATLANTIC1
}

\author{
D. Graham Jenkins, Department of Earth Sciences, Open University, Milton Keynes, United Kingdom²
}

\begin{abstract}
Thirty-one core-catcher samples from the middle Eocene to middle Miocene at Site 608 and 13 core-catcher samples from the lower to middle Miocene of Site 610 have been examined for planktonic foraminifers. Stratigraphic ranges have been established at both sites and the sequence divided into zones. Zonal markers and other datum events are correlated with the most recent time scale.
\end{abstract}

\section{INTRODUCTION}

This chapter is a shore-laboratory report on the planktonic foraminifers extracted from cores taken at Sites 608 and 610 on Leg 94 during July and August 1983. Both sites (Fig. 1) are located in the modern planktonic foraminiferal Transitional Faunal Province (Bé, 1977); their coordinates are given in the following table:

\begin{tabular}{cccc}
\hline Site & Latitude & Longitude & $\begin{array}{c}\text { Water } \\
\text { depth } \\
(\mathrm{m})\end{array}$ \\
\hline 608 & $42^{\circ} 50.21^{\prime} \mathrm{N}$ & $23^{\circ} 05.25^{\prime} \mathrm{W}$ & 3526 \\
610 & $53^{\circ} 13.30^{\prime} \mathrm{N}$ & $18^{\circ} 53.21^{\prime} \mathrm{W}$ & 2417 \\
\hline
\end{tabular}

\section{PROCEDURES}

Core-catcher sediment samples from the two sites were examined and the planktonic foraminifers identified. Semiquantitative assessments of each species were plotted on stratigraphic range charts (Tables 1,2) and the zones and epoch boundaries assigned (Figs. 2, 3).

Previous relevant work on the North Atlantic includes DSDP Leg 12 (Berggren, 1972; Poore and Berggren, 1974, 1975a, 1975b); DSDP Leg 49 (Poore, 1979); and DSDP Leg 81 (Huddlestun, 1985).

\section{EPOCH BOUNDARY MARKERS}

The Eocene/Oligocene boundary at Site 608 is marked by the extinctions of Globigerinatheka index, Globigerina linaperta, and Globorotalia cerroazulensis. An unconformity, with the lower Oligocene missing, appears to be at or near the boundary, as indicated by the planktonic foraminifers studied at a resolution of one sample per core. Uppermost Eocene Sample 608-49,CC contains an overlap of G. cerroazulensis and Globigerina ampliapertura; the latter occurs in the upper part of the G. cerroazulensis Zone (Toumarkine and Luterbacher, 1985).

The Oligocene/Miocene boundary is always difficult to identify because it is ill-defined. It has been placed at

\footnotetext{
${ }^{1}$ Ruddiman, W. F., Kidd, R. B., Thomas, E., et al., Init. Repts. DSDP, 94: Washington (U.S. Govt. Printing Office).

2 Address: Department of Earth Sciences, Open University, Milton Keynes, MK7 6AA, Buckinghamshire, United Kingdom.
}

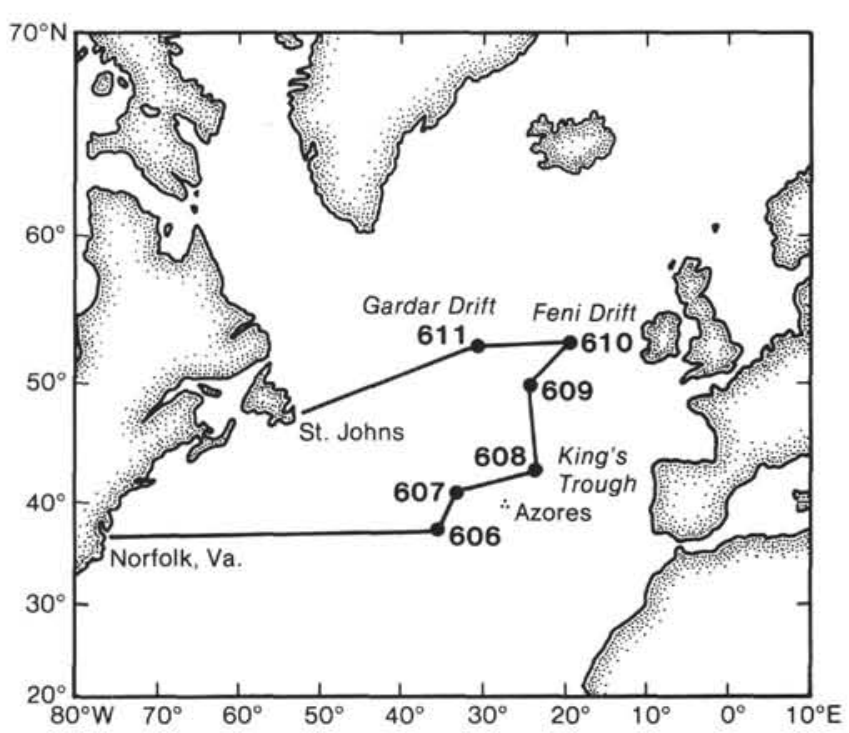

Figure 1. Map showing locations drilled on Leg 94.

Site 608 at the first appearance of Globoquadrina dehiscens, within the stratigraphic ranges of Globorotalia kugleri and Globigerinoides primordius.

\section{ZONAL SCHEMES}

Three different attempts have previously been made to subdivide the North Atlantic Eocene-Pleistocene (Berggren, 1972; Poore, 1979; and Huddlestun, 1985). Berggren (1972) established some zones for the Cenozoic of the North Atlantic on DSDP Leg 12. These were rather ill-defined, and instead of using these zones, Poore (1979), on DSDP Leg 49, attempted to assign "P" and "N" zones that Blow (1969) had established for the TropicalSubtropical Faunal Province. Huddlestun (1985) devised 13 numbered biostratigraphic intervals from the lower Eocene to the Holocene on DSDP Leg 81.

The zonal schemes used for the Eocene to middle Miocene at Site 608 and lower to middle Miocene at Site 610 are shown in Figures 2 and 3. In Figure 4, datum planes are correlated with the most recent time scale (Berggren et al., 1985). 
Table 1. Range chart with diversity data for core-catcher samples from Hole 608 .

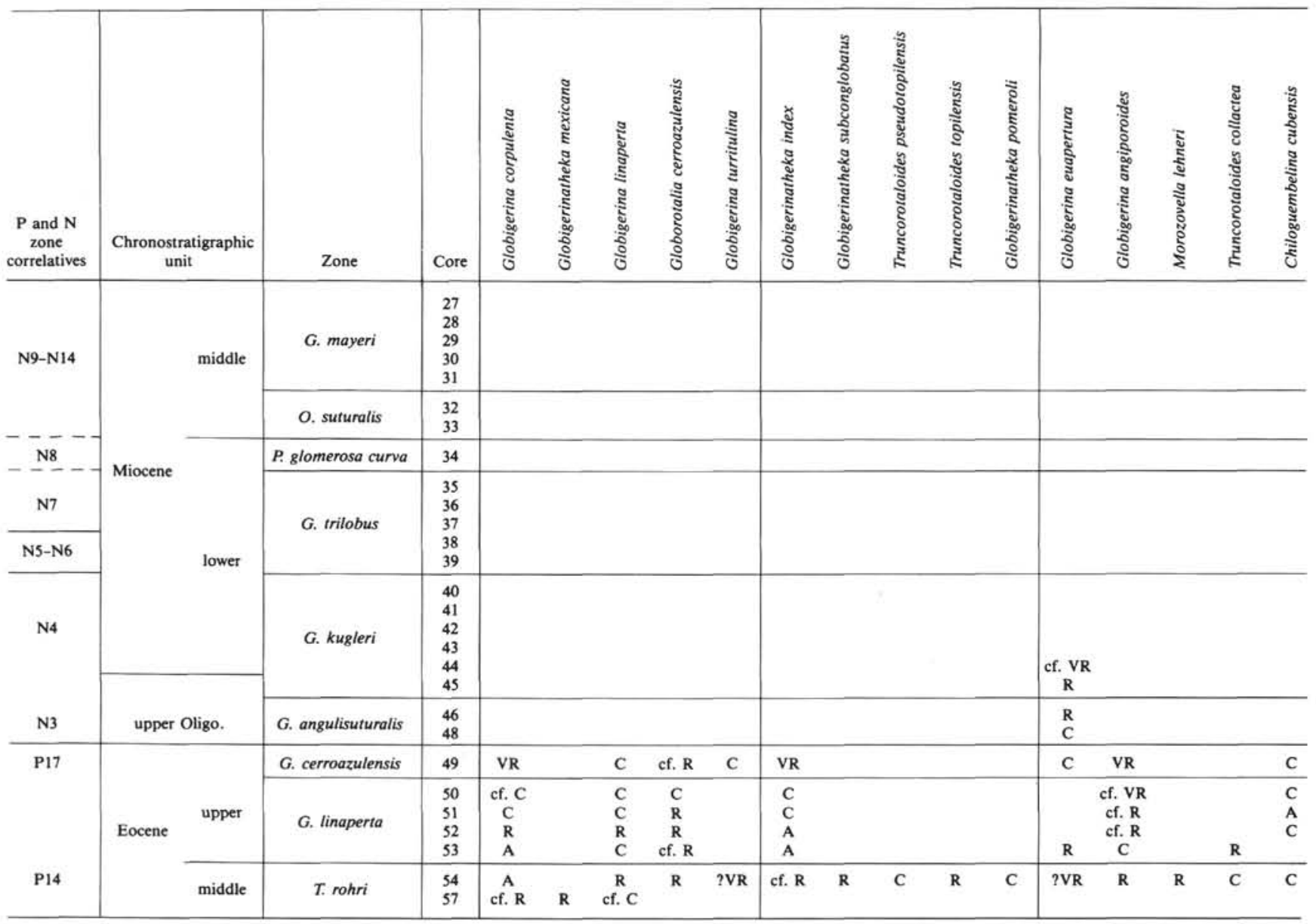

Note: $V R=$ very rare, $\mathrm{R}=$ rare, $\mathrm{C}=$ common, $\mathrm{A}=$ abundant, $\mathrm{X}=$ reworked, $\mathrm{cf} .=$ compare. Diversity $=$ number of species per sample.

\section{CONCLUSION}

A more detailed analysis of North Atlantic Cenozoic planktonic foraminifers is being undertaken to develop a more acceptable set of zonal schemes for the area.

\section{ACKNOWLEDGMENTS}

J. M. Jenkins helped in checking the manuscript, which was typed by C. Whale; J. Taylor drafted the tables.

\section{REFERENCES}

Bé, A. W. H., 1977. An ecological, zoogeographical and taxonomic review of Recent planktonic foraminifera. In Ramsey, A. T. S. (Ed.), Oceanic Micropaleontology (Vol. 1): London (Academic Press), 188.

Berggren, W. A., 1972. Cenozoic biostratigraphy and paleobiology of the North Atlantic. In Laughton, A. S., Berggren, W. A., et al., Init. Repts. DSDP, 12: Washington (U.S. Govt. Printing Office), 965-999.

Berggren, W. A., Kent, D. V., and Van Couvering, J., 1985. Neogene geochronology and chronostratigraphy. In Snelling, N. J. (Ed.), Geochronology and the Geologic Time Scale. Spec. Pap. Geol. Soc. London, 10:211-260.
Blow, W. H., 1969. Late middle Eocene to Recent planktonic foraminiferal biostratigraphy. In Brönnimann, P., and Renz, H. H. (Eds.), Proc. First Planktonic Conf.: Leiden (E. J. Brill), pp. 199-422.

Huddlestun, P. F., 1985. Planktonic foraminiferal biostratigraphy, Deep Sea Drilling Project Leg 81. In Roberts, D. G., Schnitker, D., et al., Init. Repts. DSDP, 81: Washington (U.S. Govt. Printing Office), 429-438.

Poore, R. Z., 1979. Oligocene through Quaternary planktonic foraminiferal biostratigraphy of the North Atlantic: DSDP Leg 49. In Luyendyk, B. P., Cann, J. R., et al., Init. Repts. DSDP, 49: Washington (U.S. Govt. Printing Office), 447-517.

Poore, R. Z., and Berggren, W. A., 1974. Pliocene biostratigraphy of the Labrador Sea: Calcareous plankton. J. Foram. Res., 4:91-108. , 1975a. Late Cenozoic planktonic foraminiferal biostratigraphy and paleoclimatology of Hatton-Rockall Basin: DSDP Site 116. J. Foram. Res., 5:270-293.

1975b. The morphology and classification of Neogloboquadrina atlantica (Berggren). J. Foram. Res., 5:77-84.

Toumarkine, M., and Luterbacher, H., 1985. Paleocene and Eocene planktonic foraminifera. In Bolli, H. M., Saunders, J., and PerchNielson, K. (Eds.), Plankton Stratigraphy: Cambridge (Cambridge University Press), pp. 87-154.

Date of Initial Receipt: 5 November 1984

Date of Acceptance: 19 April 1985 
MIDDLE EOCENE TO MIOCENE PLANKTONIC FORAMINIFERS

Table 1 (continued).

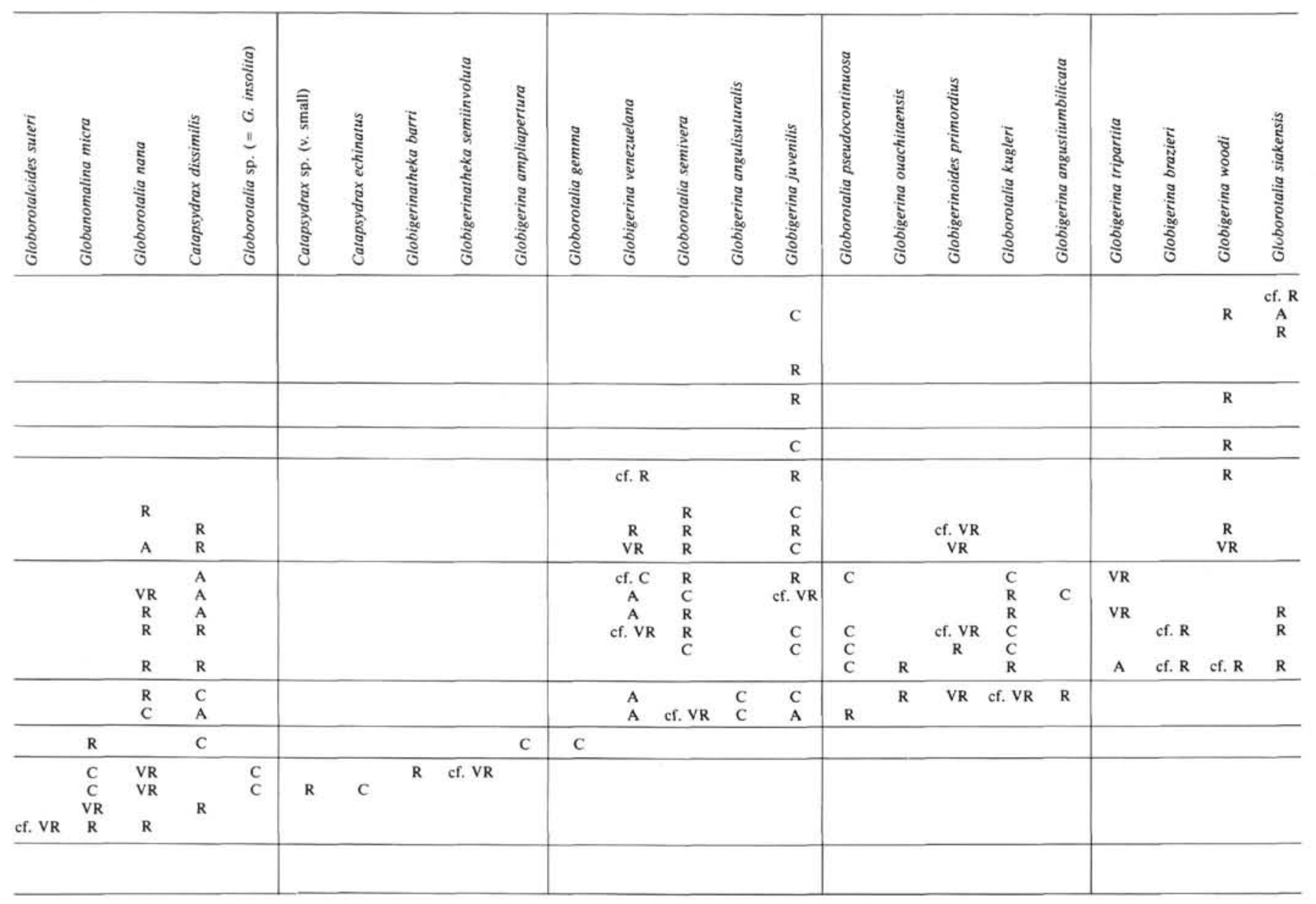

607 
D. G. JENKINS

Table 1 (continued).

\begin{tabular}{|c|c|c|c|c|c|c|c|c|c|c|c|c|c|c|c|c|c|c|c|}
\hline $\begin{array}{l}\mathrm{P} \text { and } \mathrm{N} \\
\text { zone } \\
\text { correlatives }\end{array}$ & $\begin{array}{l}\text { Chronost } \\
\text { un }\end{array}$ & tigraphic & Zone & Core & 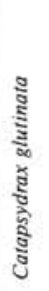 & 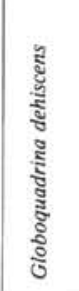 & 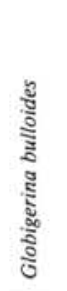 & 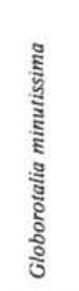 & 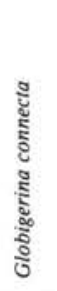 & 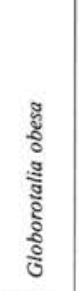 & 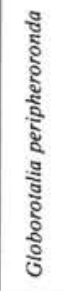 & 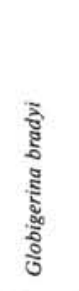 & 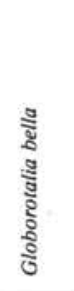 & 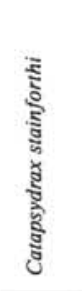 & 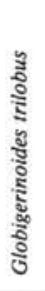 & 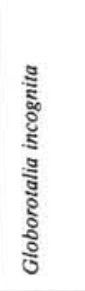 & 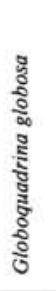 & 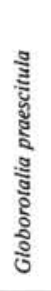 & 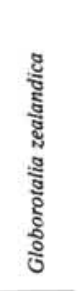 \\
\hline \multirow[t]{2}{*}{$\mathrm{N} 9-\mathrm{N} 14$} & \multirow{5}{*}{ Miocene } & \multirow[t]{2}{*}{ middle } & G. mayeri & $\begin{array}{l}27 \\
28 \\
29 \\
30 \\
31 \\
\end{array}$ & $\begin{array}{l}\mathrm{C} \\
\mathrm{C} \\
\mathrm{R} \\
\mathrm{C}\end{array}$ & $\begin{array}{l}\mathrm{C} \\
\mathrm{C} \\
\mathrm{R} \\
\mathrm{R} \\
\mathrm{A} \\
\end{array}$ & $\begin{array}{c}\text { C } \\
\text { R } \\
\text { cf. } \mathbf{R}\end{array}$ & & & $\mathbf{R}$ & & R & & & $\begin{array}{l}\mathrm{R} \\
\mathrm{C} \\
\mathrm{C} \\
\mathrm{R}\end{array}$ & & & & \\
\hline & & & O. suturalis & $\begin{array}{l}32 \\
33 \\
\end{array}$ & $\begin{array}{l}\mathrm{R} \\
\mathrm{R}\end{array}$ & $\begin{array}{l}\text { A } \\
\text { C }\end{array}$ & R & & & $\mathbf{R}$ & $\begin{array}{c}\mathrm{R} \\
\text { cf. } \mathrm{C}\end{array}$ & R & & & $\begin{array}{l}\mathrm{A} \\
\mathrm{R}\end{array}$ & & & & \\
\hline \multirow{2}{*}{$-\frac{\text { N8 }}{\text { N7 }}$} & & \multirow{3}{*}{ lower } & P. glomerosa curva & 34 & $\mathrm{c}$ & $\mathrm{c}$ & c & & & & & & & & $\mathbf{R}$ & & & $\mathrm{C}$ & \\
\hline & & & G. trilobus & $\begin{array}{l}35 \\
36 \\
37 \\
38 \\
39 \\
\end{array}$ & $\begin{array}{l}\mathbf{R} \\
\mathrm{C} \\
\mathrm{R} \\
\mathrm{C} \\
\mathrm{C}\end{array}$ & $\begin{array}{l}\mathrm{A} \\
\mathrm{A}\end{array}$ & $\begin{array}{l}\mathrm{VR} \\
\mathrm{R} \\
\mathrm{R}\end{array}$ & $\begin{array}{l}\mathrm{C} \\
\mathrm{R}\end{array}$ & & $\begin{array}{c}\mathrm{R} \\
\text { cf. } \mathrm{VR}\end{array}$ & \begin{tabular}{|c|} 
VR \\
R \\
cf. VR
\end{tabular} & $\begin{array}{l}R \\
R \\
\end{array}$ & $\begin{array}{c}\mathbf{R} \\
\mathrm{R} \\
\text { cf. } \mathrm{R}\end{array}$ & cf. VR & $\begin{array}{l}\mathrm{C} \\
\mathrm{C} \\
\mathrm{C} \\
\mathrm{C} \\
\mathrm{R}\end{array}$ & $\begin{array}{l}\text { cf. VR } \\
\text { cf. VR }\end{array}$ & $\begin{array}{l}\mathrm{C} \\
\mathrm{R} \\
\mathrm{C}\end{array}$ & $\begin{array}{l}\mathrm{C} \\
\mathrm{C} \\
\mathrm{C} \\
\mathrm{C}\end{array}$ & cf. VR \\
\hline \multirow[t]{2}{*}{$\mathrm{N} 4$} & & & G. kugleri & $\begin{array}{l}40 \\
41 \\
42 \\
43 \\
44 \\
45 \\
\end{array}$ & $\begin{array}{l}\mathrm{R} \\
\mathrm{R} \\
\mathrm{C} \\
\mathrm{C} \\
\mathrm{C} \\
\mathrm{R}\end{array}$ & $\begin{array}{l}\text { A } \\
\text { A } \\
\mathrm{C} \\
\mathrm{C} \\
\text { cf. VR }\end{array}$ & $\begin{array}{c}\text { cf. } \mathrm{R} \\
\mathrm{R} \\
\mathrm{R} \\
\mathrm{R}\end{array}$ & $\begin{array}{c}\text { cf. } V R \\
R \\
\text { ef. } R\end{array}$ & & ef. $V R$ & VR & $\begin{array}{l}\text { cf. VR } \\
\text { R } \\
\text { cf. VR }\end{array}$ & & & & & & & \\
\hline & \multicolumn{2}{|c|}{ upper Oligo. } & G. angulisuturalis & $\begin{array}{l}46 \\
48 \\
\end{array}$ & & & & & & & & & & & & & & & \\
\hline \multirow[t]{2}{*}{ P17 } & \multirow{3}{*}{ Eocene } & & G. cerroazulensis & 49 & & & & & & & & & & & & & & & \\
\hline & & upper & G. linaperta & $\begin{array}{l}50 \\
51 \\
52 \\
53 \\
\end{array}$ & & & & & & & & & & & & & & & \\
\hline P14 & & middle & T. rohri & $\begin{array}{l}54 \\
57\end{array}$ & & & & & & & & & & & & & & & \\
\hline
\end{tabular}


Table 1 (continued).

\begin{tabular}{|c|c|c|c|c|c|c|c|c|c|c|c|c|c|c|c|c|c|c|c|c|c|c|c|}
\hline 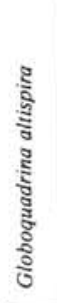 & 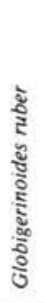 & 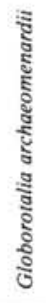 & 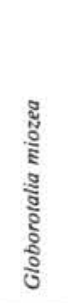 & 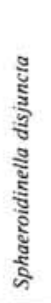 & 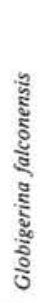 & 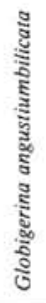 & 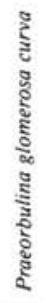 & 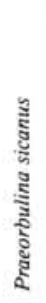 & 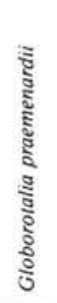 & 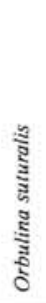 & 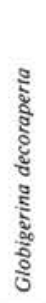 & 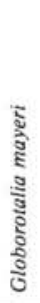 & 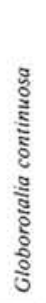 & 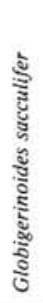 & 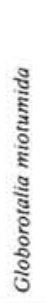 & 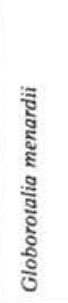 & 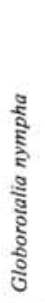 & 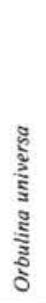 & 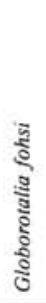 & 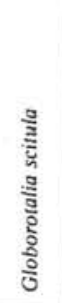 & 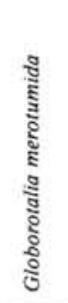 & 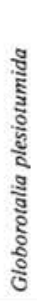 & 耪 \\
\hline $\begin{array}{l}\mathrm{R} \\
\mathrm{R} \\
\mathrm{R} \\
\mathrm{R}\end{array}$ & $\begin{array}{l}\mathrm{R} \\
\mathrm{C}\end{array}$ & & cf. $R$ & $\begin{array}{c}\mathrm{R} \\
\mathrm{R} \\
\mathrm{VR}\end{array}$ & $\begin{array}{l}\mathrm{C} \\
\mathbf{R} \\
\mathbf{R}\end{array}$ & & & & $\begin{array}{l}\mathbf{R} \\
\mathbf{C}\end{array}$ & $\begin{array}{c}\text { VR } \\
\text { R }\end{array}$ & C & $\begin{array}{l}R \\
R \\
C \\
R \\
C\end{array}$ & $\begin{array}{l}\mathrm{R} \\
\mathrm{A} \\
\mathrm{C} \\
\mathrm{C}\end{array}$ & $\mathbf{R}$ & $\begin{array}{c}\mathrm{R} \\
\mathrm{R} \\
\mathrm{R} \\
\mathrm{VR}\end{array}$ & $\begin{array}{l}\mathrm{C} \\
\mathrm{C} \\
\text { cf. } \mathrm{R} \\
\text { cf. } \mathrm{R}\end{array}$ & $\mathrm{R}$ & $\begin{array}{l}\mathrm{R} \\
\mathrm{C}\end{array}$ & $\mathbf{R}$ & ef. C & cf. $R$ & $R$ & $\begin{array}{l}15 \\
15 \\
12 \\
12 \\
13\end{array}$ \\
\hline $\begin{array}{c}R \\
V R \\
\text { VR }\end{array}$ & & C & C & & & & & & & $\begin{array}{c}R \\
V R\end{array}$ & & & & & & & & & & & & & $\begin{array}{r}13 \\
6 \\
\end{array}$ \\
\hline C & $\mathbf{R}$ & & & & R & & $R$ & $R$ & cf. $\mathbf{R}$ & & & & & & & & & & & & & & 13 \\
\hline \multirow[t]{6}{*}{$\begin{array}{l}\mathrm{C} \\
\mathrm{C} \\
\mathrm{R}\end{array}$} & C & $\mathrm{R}$ & ef. $R$ & $\mathrm{R}$ & C & $R$ & & & & & & & & & & & & & & & & & $\begin{array}{l}13 \\
11 \\
13 \\
14 \\
16\end{array}$ \\
\hline & & & & & & & & & & & & & & & & & & & & & & & $\begin{array}{r}12 \\
11 \\
13 \\
15 \\
9 \\
11\end{array}$ \\
\hline & & & & & & & & & & & & & & & & & & & & & & & $\begin{array}{r}10 \\
8\end{array}$ \\
\hline & & & & & & & & & & & & & & & & & & & & & & & 12 \\
\hline & & & & & & & & & & & & & & & & & & & & & & & $\begin{array}{r}11 \\
11 \\
8 \\
10 \\
\end{array}$ \\
\hline & & & & & & & & & & & & & & & & & & & & & & & $\begin{array}{r}14 \\
3\end{array}$ \\
\hline
\end{tabular}


Table 2. Range chart with diversity data for core-catcher samples from Hole 610.

\begin{tabular}{|c|c|c|c|c|c|c|c|c|c|c|c|c|c|c|c|c|c|c|c|c|c|c|c|c|}
\hline $\begin{array}{l}\text { Chrono- } \\
\text { stratigraphic } \\
\text { unit }\end{array}$ & Zone & Core & 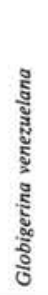 & 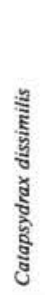 & 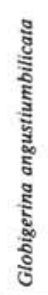 & 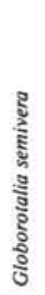 & 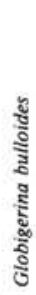 & 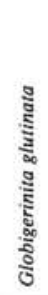 & 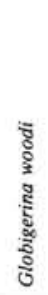 & 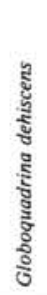 & 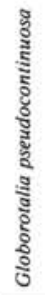 & 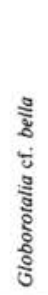 & 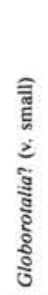 & 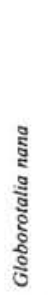 & 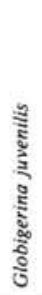 & 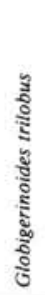 & 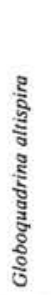 & 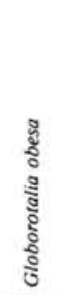 & 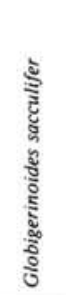 & 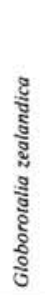 & 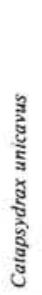 & 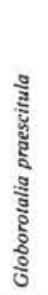 & 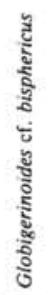 & $\begin{array}{l}\text { ัूँ } \\
\text { है } \\
\text { gूँ }\end{array}$ \\
\hline u. Mio. & N. humerosa & 15 & & & & & A & $\mathrm{R}$ & & $\mathrm{R}$ & & & & & c & & $\mathbf{R}$ & & & & & & & \\
\hline m. Mio. & G. mayeri & 16 & & & & & $\mathrm{c}$ & $\mathrm{R}$ & & $\mathrm{R}$ & & & & & & $\mathbf{R}$ & & VR & & & & & & \\
\hline \multirow{3}{*}{$\begin{array}{c}\text { lower } \\
\text { Miocene }\end{array}$} & G. glomerosa curva & 17 & & & $\mathrm{C}$ & & $\mathrm{C}$ & & & $\mathbf{R}$ & & & & & & A & $\mathbf{R}$ & VR & VR & & & VR & & \\
\hline & G. trilobus & $\begin{array}{l}18 \\
19 \\
20 \\
21 \\
22 \\
23\end{array}$ & A & $\mathrm{R}$ & & $\begin{array}{l}\mathrm{R} \\
\mathrm{R}\end{array}$ & $\begin{array}{l}\mathrm{C} \\
\mathrm{C} \\
\mathrm{C} \\
\mathrm{C} \\
\mathrm{C}\end{array}$ & c & $\begin{array}{c}\mathrm{R} \\
\mathrm{C} \\
\mathrm{VR}\end{array}$ & $\begin{array}{l}\mathrm{A} \\
\mathrm{A} \\
\mathrm{A} \\
\mathrm{R} \\
\mathrm{R} \\
\mathrm{R}\end{array}$ & $\begin{array}{l}\mathrm{R} \\
\mathrm{C}\end{array}$ & & & & $\mathrm{R}$ & $\begin{array}{l}\mathrm{R} \\
\mathrm{C} \\
\mathrm{R} \\
\mathrm{R} \\
\mathrm{C} \\
\mathrm{C}\end{array}$ & $\begin{array}{c}\mathrm{C} \\
\text { VR } \\
\mathrm{R}\end{array}$ & cf. $R$ & cf. $\mathbf{R}$ & C & A & $\begin{array}{l}\mathrm{C} \\
\mathrm{C} \\
\mathrm{C} \\
\mathrm{C}\end{array}$ & $\begin{array}{c}R \\
\text { VR } \\
\text { VR }\end{array}$ & $\begin{array}{l}R \\
C\end{array}$ \\
\hline & C. dissimilis & $\begin{array}{l}24 \\
25 \\
26 \\
27\end{array}$ & $\begin{array}{l}\mathrm{C} \\
\mathrm{C} \\
\mathrm{A}\end{array}$ & $\begin{array}{l}\mathrm{R} \\
\mathrm{R} \\
\mathrm{C} \\
\mathrm{C}\end{array}$ & VR & $\begin{array}{c}\mathrm{R} \\
\mathrm{R} \\
\mathrm{VR}\end{array}$ & $\begin{array}{l}\mathrm{C} \\
\mathrm{C} \\
\mathrm{C} \\
\mathrm{C}\end{array}$ & $\begin{array}{l}\text { VR } \\
\text { VR } \\
\text { R }\end{array}$ & $\begin{array}{c}\text { VR } \\
R \\
\text { VR }\end{array}$ & $\begin{array}{c}\text { C } \\
\text { C } \\
\text { A } \\
\text { VR }\end{array}$ & $\begin{array}{l}\text { C } \\
\mathrm{C} \\
\mathrm{C} \\
\mathrm{VR}\end{array}$ & $\begin{array}{l}\text { VR } \\
\text { VR }\end{array}$ & VR & $\begin{array}{l}R \\
R \\
R\end{array}$ & $\mathbf{R}$ & & & & & & & & & \\
\hline
\end{tabular}

Note: $\mathrm{VR}=$ very rare, $\mathrm{R}=$ rare, $\mathrm{C}=$ common, $\mathrm{A}=$ abundant, $\mathrm{cf} .=$ compare. Diversity $=$ number of species per sample.

Table 2 (continued)

\begin{tabular}{|c|c|c|c|c|c|c|c|c|c|c|c|c|c|c|c|c|c|c|c|c|c|c|c|c|}
\hline $\begin{array}{l}\text { Chrono- } \\
\text { stratigraphic } \\
\text { unit }\end{array}$ & Zone & Core & 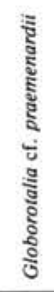 & 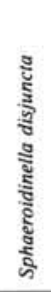 & 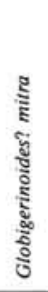 & 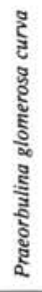 & 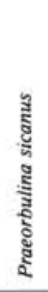 & 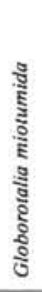 & 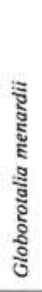 & $\begin{array}{l}\text { हैँ } \\
\text { हैँ } \\
\text { हैँ } \\
\text { हैँ }\end{array}$ & 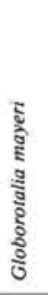 & 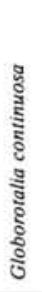 & 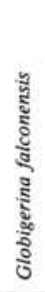 & 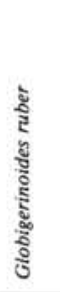 & 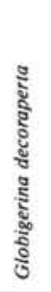 & 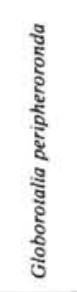 & 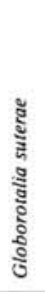 & 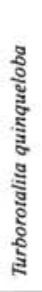 & 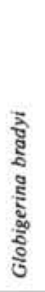 & 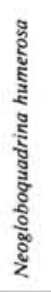 & 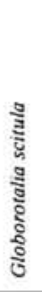 & 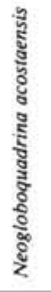 & 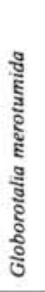 & 㐔 \\
\hline u. Mio. & N. humerosa & 15 & & VR & & & & & $R$ & & & & & . & $\mathbf{R}$ & & & $\mathbf{R}$ & $\mathrm{R}$ & $\mathrm{A}$ & $\mathrm{C}$ & $\mathrm{A}$ & $\mathrm{c}$ & 14 \\
\hline m. Mio. & G. mayeri & 16 & & & & & & $\mathrm{C}$ & $R$ & $\mathrm{R}$ & A & $\mathrm{R}$ & VR & VR & VR & ef. VR & $\mathrm{C}$ & $\mathrm{C}$ & $\mathrm{C}$ & & & & & 17 \\
\hline \multirow{3}{*}{$\begin{array}{c}\text { lower } \\
\text { Miocene }\end{array}$} & G. glomerosa curva & 17 & & & & $\mathbf{R}$ & VR & & & & & & & & & & & & & & & & & 10 \\
\hline & G. trilobus & $\begin{array}{l}18 \\
19 \\
20 \\
21 \\
22 \\
23\end{array}$ & vR & c & $\mathrm{R}$ & & & & & & & & & & & & & & & & & & & $\begin{array}{r}8 \\
8 \\
7 \\
8 \\
7 \\
12 \\
\end{array}$ \\
\hline & C. dissimilis & $\begin{array}{l}24 \\
25 \\
26 \\
27\end{array}$ & & & & & & & & & & & & & & & & & & & & & & $\begin{array}{r}7 \\
8 \\
10 \\
11\end{array}$ \\
\hline
\end{tabular}




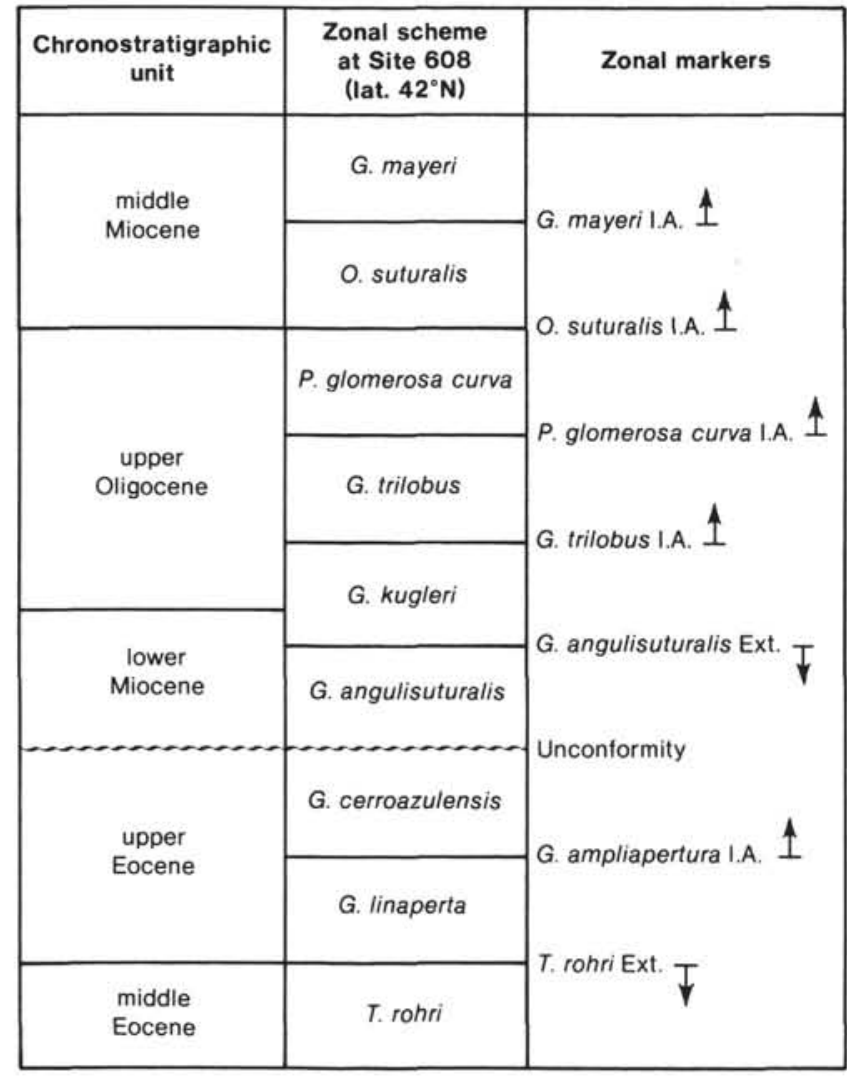

Figure 2. Zones and zonal markers at Site 608. I.A. = initial appearance, Ext. = extinction.

\begin{tabular}{|c|c|c|}
\hline $\begin{array}{c}\text { Chronostratigraphic } \\
\text { unit }\end{array}$ & $\begin{array}{l}\text { Zonal scheme } \\
\text { at Site } 610 \\
\text { (lat. } 53^{\circ} \mathrm{N} \text { ) }\end{array}$ & Zonal markers \\
\hline $\begin{array}{l}\text { middle } \\
\text { Miocene }\end{array}$ & G. mayeri & \multirow{4}{*}{$\begin{array}{l}\text { G. mayeri I.A. I } \\
\text { P. glomerosa curva I.A. } 1 \\
\text { G. trilobus I.A. } 1\end{array}$} \\
\hline \multirow{3}{*}{$\begin{array}{l}\text { lower } \\
\text { Miocene }\end{array}$} & P. glomerosa curva & \\
\hline & G. trilobus & \\
\hline & C. dissimilis & \\
\hline
\end{tabular}

Figure 3. Zones and zonal markers at Site 610. I.A. = initial appearance, Ext. = extinction.

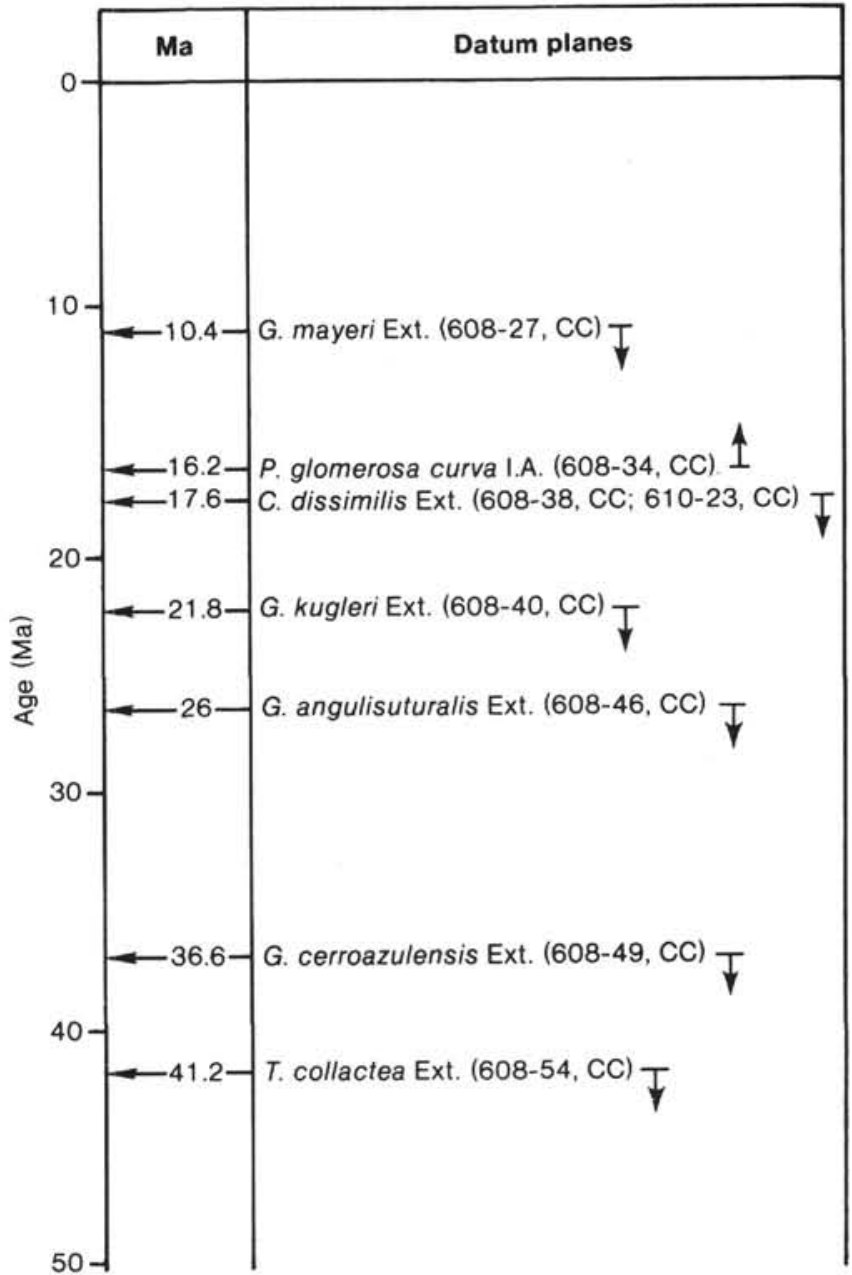

Figure 4. Site data and datum planes correlated with the Berggren et al. (1985) time scale. I.A. = initial appearance, Ext. = extinction. 